\title{
PHYSICS AND CHEMISTRY OF CERAMIC SURFACES
}

$\mathrm{A}^{\mathrm{N}}$ interesting and successful symposium on ceramic surfaces was organized recently by the Basic Science Section of the British Ceramic Society. The meeting, held in London during December 15-17, 1964, attracted scientists with diverse interests. Among the audience were research workers active in many different branches of ceramic science-porosity and texture of oxides, gas adsorption, electron microscopy, irradiation phenomena and the mechanical properties of solids, to mention but a few. The fact that such varied interests were represented, particularly at a symposium held just one week before Christmas, is surely a reflexion of the importance of surface physics and chemistry in ceramic rosearch to-day.

The symposium was opened by the honorary president, Sir Eric Rideal, and the introductory lecture was delivered by Prof. J. H. de Boer. Prof. de Boer's theme was the change in pore shapes and pore systems brought about by sintering at temperatures well below the Tammann temperature. Such low-temperature sintering may be identified, and its nature characterized, from studies of nitrogen physical absorption isotherms. The method is based on the observation that for all flat surfaces, free from capillaries, the adsorption isotherm has a unique form. Hence a plot of gas adsorbed versus thickness $(t)$ of the adsorbed film is linear, with a slope proportional to the surface area of the absorbent. Deviations from linearity are of two types: an upward bending of the plot indicates capillary condensation, while a downward curvature is interpreted as the filling of slit-shaped crevices by multi-layer adsorption on parallel walls. Prof. de Boer has applied this concept to the study of pore systems present in the dehydration products of hydrous $\mathrm{ZrO}_{2}$ and two alumina hydrates (baverite and boehmite). For $\mathrm{ZrO}_{2}$, there is conventional capillary condensation in very fine pores, beginning at a lower relative pressure than is indicated by the hysteresis loop in the isotherm. By contrast, well crystalline bayerite and boehmite dehydrate to $\eta$ and $\gamma \mathrm{Al}_{2} \mathrm{O}_{3}$ respectively, with particles which are pseudomorphic after the original crystals and consist of parallel, oriented lamellae, separated by slit-shaped pores $\sim 10 \AA$ wide. These pores aro filled by multilayer adsorption in the pressure range $P / P_{0}=0.1-0.4$. From the change in slope of the $t$ plot the surface area is found to decrease abruptly as the pores are filled. Investigations of evaporated films of ionic salts $\left(\mathrm{NaCl}, \mathrm{CaF}_{2}\right.$, etc.) condensed on a cold substrate indicate that these too possess a similar lamellar structure.

The seventeen contributed papers could be divided broadly into two groups: (1) those concerned with the structure of crystal surfaces and the experimental examination of surfaces, and (2) those dealing with the adsorption of gases, particularly water. In the first group, Dr. W. J. Dunning (University of Bristol) gave an interesting treatment of crystal surfaces from a theoretical point of view. He considered a real surface as possessing certain idealized defects-vacancies, steps, kink sites and emergent dislocations. The adsorption of a foreign atom at these various sites leads to a change in surface roughness. For a simple cubic, homopolar crystal, this change in roughness may be calculated knowing the interaction energy between the adsorbate atom and atoms in the crystal surface. Thermal fluctuations tend to restore the equilibrium density of imperfections in the crystal surface. For ionic crystals containing transition metal ions, it is necessary to consider the influence of the asymmetric crystal field at the surface on the $d$ orbitals of the cations. In general, crystal field forces may be expected to lead to a relaxation in the positions of surface ions.
In an experimental investigation of the surfaces of alkali bromide crystals, L. W. Barr and D. K. Dawson (Harwell) have investigated the exchange reaction between inactive bromine vapour and labelled $\mathrm{KBr}$ crystals containing ${ }^{82} \mathrm{Br}^{-}$ions produced by neutron irradiation. The surface exchange reaction was readily distinguished from bulk phase exchange. There is some evidence that $\mathrm{Br}_{3}{ }^{-}$ ions are formed as an activated complex. Electron microscopy was used by A. J. Forty and C. T. Forwood (University of Bristol) in a study of alkali halides and by P. S. Maxwell and D. J. Harwood (General Electric Co., Wembley) in an examination of devitrified glass ceramies. Crystals of sodium chloride containing voids and precipitates may be cleaved and the cleavage surface examined using a decorated replica technique. Interesting and characteristic cleavage steps are observed in the region surrounding inhomogeneities. These steps are produced by localized plastic deformations, induced by the travelling crack when impeded by inhomogeneities. This localized interruption of cleavage, with the consequent development of steps, affects the propagation of a crack through the entire crystal. The possibility of 'crack stopping' by the incorporation of suitable inclusions clearly merits further investigation. The examination of devitrified glass ceramics, also using a replica technique, revealed the presence of a high concentration of fine pores, up to $100 \AA$ in size. These are believed to be present in the glass before devitrification. The cleavage of polycrystalline ceramics ('brittle fracture') was investigated optically by V. D. Frechette (Now York State College of Ceramics), who illustrated the many possible modes of fracture for different ceramic materials. One is left with the impression that there is much to be learnt from a careful examination and analysis of fracture surfaces. Finally, in this group of papers, C. F. Cooper (Morganite Research, Wandsworth) discussed the surface behaviour of molten silicates. In $\mathrm{CaO}-\mathrm{SiO}_{2}$ melts there is a preferential concentration of silica at the surface. This may be interpreted in terms of the Gibbs adsorption isotherm, with $\mathrm{SiO}_{2}$ possessing a lower surface energy than $\mathrm{CaO}$.

Turning to the papers concerned with the adsorption of gases on ceramic surfaces, Prof. R. M. Barrer (Imperial College of Science and Technology) presented a comprehensive review of surface flow phenomena in gases and vapours physically adsorbed on micro-porous, highsurface-area materials. Results for a wide variety of adsorbates and adsorbents were summarized, and it was concluded that measurements of surface diffusion can be related to the adsorption properties of a gas and, furthermore, can provide information on the texture of a porous adsorbent. Another topic to be reviewed critically was the determination of specific surface areas by low-temperature krypton adsorption. In this paper, K. S. W. Sing and D. Swallow (College of Technology, Liverpool) demonstrated that the shape of the isotherm depends on the uniformity and porosity of the surface and that the Brunauer-Emmett-Teller equation provides a reliable estimate of the surface-area only under specific conditions. One should, however, recall that in introducing the krypton surface area technique in 1945, Prof. Beebe limited its application to low-surface-area non-porous solids, for which its practical utility has since been amply demonstrated.

Six papers altogether were concerned with water adsorption on oxides. Two of these discussed the interaction of water with silica surfaces, a problem which has been extensively studied with only partial agreement being reached. W. K. Thompson (Unilever, Port Sunlight) reported infra-red absorption spectra for water physically adsorbed on porous silica; from his data he concludes that 
the water is hydrogen-bonded both intermolecularly and to hydroxyl groups on the $\mathrm{SiO}_{2}$ surface. A new approach to this problem was provided by J. A. Hockey et al. (Manchester College of Science and Technology), who measured the heats of immersion of dehydrated silica in water. From the results they deduced that the distribu. tion of residual $\mathrm{OH}$ groups on a dehydrated silica surface depends on the conditions of outgassing; sintering in air facilitates redistribution of the $\mathrm{OH}$ groups to sites of lowest energy. Two other papers, both by P. J. Anderson and colleagues (Harwell), were devoted to water adsorption on magnesia, an oxide which has been much less studied in this context. Infra-red absorption measurements revealed the surface dehydration-hydration cycle not to be reversible; rather it seems that dehydration leads to a small change in the surface ionic arrangement such that subsequent rehydration occurs to form hydroxyl groups which are bonded differently. This general picture is confirmed and amplified by proton magnetic resonance studies of ehemisorbed and physically absorbed water on MgO. These measurements suggest that hydruxyl groups on a partially dehydrated surface may form small clusters. The application of nuclear magnetic resonance to surface chemistry is of recent origin and promises to be a powerful analytical tool for selected problems in adsorption. The remaining papers on water adsorption were by S. J. Gregg et al. (University of Exeter) and by D. Hardy and N. J. Petch (University of Newcastle). Gregg presented water adsorption isotherms for a number of oxides, $\mathrm{Fe}_{2} \mathrm{O}_{3}, \mathrm{SnO}_{2}, \mathrm{SiO}_{2}$ and $\mathrm{TiO}_{2}$. Silica is particularly interesting as it is hydrophobic after calcination at $900^{\circ} \mathrm{C}$, but slowly regains its hydrophilic nature during prolonged exposure to water vapour at $25^{\circ} \mathrm{C}$. Hardy and Petch in their paper considered the lowering in the surface energy of $\gamma$-and $\alpha$-alumina brought about by water adsorption.
The experimental decrease in surface energy, calculated from adsorption isotherms, is quite insufficient to account for the reduction in fracture stress of $\mathrm{Al}_{2} \mathrm{O}_{3}$ observed in the presence of water vapour.

The final afternoon session comprised three miscellaneous papers each interesting in its own way. R. A. Ross and A. H. Taylor (College of Technology, Belfast) extended their earlier work on ammonia adsorption on silica gel with a new study of ethylamine adsorption. R. L. Nelson et al. (Harwell) discussed the influence of irradiation on the chemisorption of oxygen on $\mathrm{MgO}$ and $\mathrm{CaO}$. Electron spin resonance techniques were exploited to provide information on irradiation-induced surface defects and their role in chemisorption. Finally, J. K. Higgins (Harwell) provided a beautiful illustration of how a technologically oriented study may lead to results of considerable scientific interest. In an investigation of the reaction of alumina with caesium vapour, neutron irradiation was found to enhance the reaction considerably. The effect was attributed to minor cracking in the alumina, resulting from irradiation-induced lattice expansion.

From the foregoing brief account it will be seen that the symposium, while directed towards a central theme, embraced papers which were concerned with a wide variety of experimental techniques and with diverse types of ceramic material. This follows a pattern set by recent meetings of the Basic Science Section of the British Ceramic Society. Such meetings are especially valuable in fostering an exchange of ideas between scientists drawn from different disciplines and industries but united by a common interest. From the lively discussion which developed, one may conclude that the present symposium succeeded in this aim. Much of the credit for this success must go to the organizer, Dr. P. J. Anderson.

R. M. Deur

\section{ANIMAL AND PLANT TISSUE CULTURE}

\begin{abstract}
A SEMINAR on animal and plant tissue culture was held at the Department of Biochemistry, M.S. University of Baroda, Baroda, India, under the auspices of the University Grants Commission and the M.S. University of Baroda, during January 21-28. Prof. C. V. Ramkrishnan, head of the Department of Biochemistry, M.S. University of Baroda, was the director of the seminar. This was the second conference on tissue culture to be held in India, the first having been held at the Department of Botany, University of Delhi, under the auspices of the Unesco South Asia Science Co-operation Office, New Delhi, in December 1961. The latter, however, has been confined to plant tissue culture.

The present seminar was attended by about sixty delegates, drawn not only from different parts of India but also from overseas, and they included biochemists, botanists, zoologists and virologists. The overseas visitors included: Dr. C. R. Anderson (now at the Virus Research Centre, Poona); Prof. E. J. Ambrose (Chester Beatty Research Institute, London); Dr. Endo Hiroyoshi (University of Tokyo); Dame Honor B. Fell (Strangeways Research Laboratory, Cambridge); Prof. H. Katsuta (Tokyo University); Prof. J. Paul (University of Glasgow); Prof. M. D. Rosemberg (Tho Rockefeller Institute, New York); and Prof. C. H. Waddington (University of Edinburgh).
\end{abstract}

Dr. C. S. Patel, vice-chancellor, M.S. University of Baroda, welcomed the delegates, and Dame Honor B. Fell delivered the inaugural address in which she outlined the history of the development of animal tissue culture studies.

The seminar consisted of fifteen sessions: two for animal organ culture, seven for animal cell culture, two for virology, and four for plant tissue culture. In all, about
43 papers were presented, of which 25 were review papers and the remainder reported original work.

In the sessions on organ culture, special mention should be made of the two lectures given by Dame Honor $B$. Fell, one on the technique of organ culture and the other on the application of organ culture to medical and biological research. Other papers on organ culture included: ossification of chick embryo femur growing in liquid media (Prof. H. Endo et al.); incorporation of labelled phosphorus in nucleic acids of human trophoblastic tumours in organ culture (Drs. M. Shee and J. R. Chowdhury); effect of steroid hormones on the chondroitin sulphate metabolism of chick embryo femur growing in vitro (Drs. H. Endo and S. Murota); and biochemical studies on embryonic chick tibia cultivated in vitro (Drs. H. R. Chokshi and C. V. Ramkrishnan).

By far the largest number of papers were read at the animal cell culture sessions, of which the following goneral lectures were of special interest: animal cell culture in cell biology research (Prof. J. Paul); the growth of cells at saline-hydrocarbon interface (Prof. M. D. Rosemberg); carcinogenesis in tissue culture (Prof. H. Katsuta); malignancy of tumour at the cellular level (Prof. H. Katsuta); messenger RNA in animal cells (Prof. J. Paul); culture of differentiating amphibian embryonic cells (Prof. C. H. Waddington); cell contacts and cell movements using tissue culture and biophysical techniques, and the properties of the cell surface (Prof. E. J. Ambrose). Among the original papors presented at the cell culture sessions, mention may be made of the following: effect of steroid hormones on HeLa cells (Prof. H. Endo); studies on normal and cancerous human cervix tissue maintained in vitro in synthetic medium (Prof. C. V. Ramkrishnan et al..); and rat thymus cells in culture (Prof. H. Katsuta). 